\title{
Crecimiento económico y capital humano: metodología para la simulación de una variante del Modelo de Lucas con aplicación a México
}

\author{
Alejandro Rodríguez Arana* \\ Universidad Iberoamericana, Ciudad de México, Departamento de Economía
}

(Primera recepción: 26 de Enero 2016, Segunda recepción: 20 de Septiembre 2016, Aceptado: 12 de Diciembre 2016)

\begin{abstract}
Resumen
Este trabajo tiene como principal objetivo plantear una variante del modelo de crecimiento de Lucas (1988) que pueda fácilmente ser sujeta a ejercicios de calibración y simulación. Un segundo objetivo es calibrar y simular el modelo propuesto para el caso de México. Para lograr lo anterior, se parte del modelo original de Lucas (1988) y de la estructura de ahorro-inversión propuesta por Solow $(1956,1957)$. El ejercicio de calibración-simulación para México muestra que aumentar el crecimiento del producto per cápita en este país en sólo unas décimas requiere un esfuerzo de ahorro adicional difícil de generar en los próximos años, por lo cual es posible que el crecimiento del futuro cercano siga siendo bajo. La metodología propuesta puede generar un sesgo donde el crecimiento depende en exceso de la trayectoria de capital humano, eso sucede en modelos del tipo de Lucas $(1988,2009)$. Sin embargo, las formas reducidas del modelo planteado pueden calibrarse y simularse con relativa facilidad para la mayoría de los países que tienen información bases de datos públicas, como en la Penn World Table.

Clasificación JEL: I25, E21, E22, E24.

Palabras clave: Capital humano, Tiempo dedicado a la educación, Crecimiento económico.
\end{abstract}

\section{Economic Growth and Human Capital: Methodology to Simulate a Lucas Model variant with application for Mexico}

\section{Abstract}

The main objective of this paper is to set a variant of the Lucas (1988) model that may be easily calibrated and simulated. A second goal is to calibrate and simulate the proposed model for México. To achieve the previous objectives, we draw from the original Lucas (1988) model and the saving-investment structure proposed by Solow $(1956,1957)$. A calibrationsimulation exercise for México shows that in order to increase the growth of the product per capita by less than one percentage, it is necessary to generate an additional savings, which is difficult to generate in the coming years. For this reason, growth in this country quite possibly will continue being low in the following years. The proposed methodology may generate a bias where growth depends in excess of the trajectory of human capital, which happens in Lucas type models (Lucas 1988, 2009). However, the reduced forms of the proposed model may be calibrated and simulated with relative ease for the majority of the countries that have information in public databases, like the Penn World Table.

JEL Classification: I25, E21, E22, E24.

Key Words: Human Capital, Time dedicated to Education, Economic Growth.

\footnotetext{
* Prolongación Paseo de la Reforma 880, Colonia Lomas de Santa Fe, Ciudad de México, México, C. P. 01219, Teléfono 59-50-42-68, Correo electrónico: alejandro.rodriguez@ibero.mx
} 
24 Nueva Época REMEF (The Mexican Journal of Economics and Finance)

\section{Introducción}

El modelo de Solow (1956) (1957) revolucionó la teoría del crecimiento económico. Sus resultados en cuanto a convergencia condicional y el que las economías crecen en el largo plazo por el aumento de la productividad, son hoy en día clásicos. Los trabajos posteriores de Cass (1965) y Koopmans (1965), en presencia de consumidores que optimizan, confirmaron los principales resultados de Solow.

En los años ochenta, Romer (1986) y Lucas (1988) se preguntan de dónde surge la productividad en el modelo de Solow. Romer propone la hipótesis de que la productividad surge del aprender haciendo (learning by doing), concepto tiempo atrás acuñado por Arrow (1962). Lucas retoma el trabajo de Uzawa (1965) y propone que la productividad está determinada básicamente por la acumulación de capital humano. En particular, Lucas (1988) busca comprender y determinar los componentes que explican la creación de capital humano.

El modelo de Lucas (1988) encuentra que el crecimiento del capital humano está relacionado básicamente con dos factores: la calidad de la educación y el porcentaje de tiempo que las personas dedican al estudio. Si estos factores fueran exógenos, el crecimiento de la productividad descrita en el modelo de Solow (1957) estaría determinado uno a uno por los dos factores expuestos por Lucas. Sin embargo, Lucas obtiene de manera endógena el porcentaje de tiempo dedicado al estudio. Al final, el crecimiento económico termina dependiendo de factores como la tasa de preferencias intertemporales, la elasticidad de sustitución en el consumo y la tasa de depreciación del capital. ${ }^{1}$ En cambio, en el modelo de Solow (1956) (1957), y los posteriores de Cass (1965) y Koopmans (1965), el crecimiento de largo plazo depende del cambio exógeno de la productividad y no de los otros factores.

Si bien hay una gran cantidad de trabajos empíricos en el marco del modelo original de Solow (1956) (1957), ${ }^{2}$ los cuales tratan el crecimiento del capital humano como exógeno, los trabajos prácticos utilizando como referencia el modelo de Lucas son más escasos. ${ }^{3}$ La razón principal de este fenómeno pudiera ser el hecho de que obtener los parámetros básicos del modelo de Lucas (1988) no es del todo fácil. Dos de esos parámetros principales son la tasa de descuento intertemporal y la elasticidad sustitución en el consumo.

Los mencionados parámetros del párrafo anterior podrían obtenerse estimando una ecuación de Euler para el consumo. Muchos trabajos lo hacen. ${ }^{4}$ Sin embargo, hay cuando menos dos problemas con los parámetros estimados:

\footnotetext{
1 Modelo de crecimiento endógeno.

2 Ver por ejemplo Barro (1991) (2001), Mankiw, Romer y Weil (1992), Krueger y Lindahl (2001), Vandenbussche et al. (2004), Arnold et al. (2007), Cohen y Soto (2007), Aghion et al. (2010), Dao (2012), Gennaioli et al. (2013).

3 El artículo de Lucas (1988) hace una calibración para Estados Unidos, el propio Lucas (2009) vuelve a hacer una calibración de un modelo basado en su artículo de 1988. Otros trabajos que calibran modelos basados en el de Lucas (1988) son el de Bils y Klenow (2000), Shapiro (2006), Gennaioli et al. (2013), Manuelli y Seshadri (2014).

4 Por ejemplo Attanasio y Low (2002); Fuhrer y Rudebusch (2004); Fuhrer y Olivei (2004); Cooper, Haltiwanger y Willis (2010); Lewbel, Linton y Srisuma (2011) y Rodríguez (2012).
} 
El primero es que a menudo las formas puras de la ecuación de Euler no presentan una estimación econométrica adecuada (ver por ejemplo Fuhrer y Rudebusch (2004)), por lo cual hay que introducir parámetros que no están directamente indicados en la teoría. Esto puede suceder por ejemplo por la formación de hábitos. Formas alternativas de la ecuación de Euler deben entonces incluir no sólo el consumo futuro, sino uno o varios rezagos del consumo pasado. Al encontrar este problema habría que modificar el trabajo original de Lucas (1988) y eso podría ser complicado.

Un segundo problema es que a veces, aunque la ecuación de Euler tiene un buen ajuste empírico (ver por ejemplo Rodríguez (2012)), algunos de los parámetros estimados tienen una varianza tan grande que impide que su media pueda ser utilizada para llevar a cabo ejercicios de simulación. ${ }^{5}$

Por lo anterior, sería deseable simular el modelo de Lucas (1988) con parámetros observados, o que puedan ser estimados con menos problemas. Una alternativa es utilizar una versión del modelo de Solow (1957). En dicho modelo parámetros como la tasa de ahorro, la tasa de crecimiento de la población y la tasa de depreciación del capital son variables observables.

A partir de las observaciones anteriores, el objetivo principal de este trabajo es calibrar y simular el modelo de Lucas (1988) para México a través de una versión ligeramente modificada del modelo de Solow (1957). Con dicha simulación pueden obtenerse los efectos que tienen diversos parámetros sobre el crecimiento del PIB per cápita de largo plazo. ${ }^{6}$ En algunos casos esto es relevante para llevar a cabo políticas que propicien un mayor crecimiento sostenido, más en un país como México cuyo crecimiento es muy bajo desde hace más de tres décadas. Hasta donde tenemos noticia, no se ha llevado a cabo un ejercicio de este tipo para México.

Un segundo objetivo del trabajo es mostrar que las ideas centrales de Lucas (1988) no requieren necesariamente de un enfoque de optimización del consumo, sino que pueden reproducirse en situaciones donde la tasa de ahorro se determina por una formación de hábitos. La ventaja de utilizar este supuesto es práctica. Las cifras de la tasa de ahorro están disponibles para una gran cantidad de países. En cambio, parámetros como el de la elasticidad de sustitución en el consumo se tienen que estimar y, como ya se ha mencionado, dichas estimaciones no siempre son confiables.

En el análisis del crecimiento de largo plazo de un país en particular, una ventaja de utilizar la técnica de calibración-simulación en un modelo como el de Lucas (1988)- y no una metodología econométrica tradicional- es la de evitar el problema de endogeneidad. Una gran cantidad de trabajos econométricos ${ }^{7}$

\footnotetext{
5 Por ejemplo, en el trabajo de Rodríguez (2012) el estimador de la elasticidad sustitución en el consumo tiene una varianza muy pequeña, pero la varianza del estimador de la tasa de descuento intertemporal es muy grande y no es significativo.

6 Los parámetros en cuestión son: la participación del capital en la economía, la tasa de crecimiento de la población, la tasa de ahorro, la tasa de depreciación del capital físico y un indicador de la calidad de la educación.

7 Ver por ejemplo Barro (1991) (2001), Mankiw, Romer y Weil (1992), Krueger y Lindahl (2001), Vandenbussche et al. (2004), Arnold et al. (2007), Cohen y Soto (2007), Aghion et al. (2010), Dao (2012), Gennaioli et al. (2013), entre muchos otros.
} 
analiza los efectos del capital humano sobre el crecimiento. Sin embargo, los modelos tipo Lucas $(1988,2009)$ señalan con claridad que diversas variables que inciden directamente sobre el crecimiento de largo plazo también tienen un efecto sobre el capital humano. Cuando esas variables se utilizan como controles de las regresiones econométricas, se subestima el efecto total que tiene el capital humano sobre el crecimiento económico (Lucas (2009), Manuelli y Seshadri $(2014)) .^{8}$

No obstante lo anterior, modelos como los de Lucas $(1988,2009)$ también presentan limitaciones. La principal es que por la forma en la que están construidos terminan generando que en largo plazo el crecimiento del producto per cápita sea, o muy similar al del capital humano, o cuando menos proporcional a él, lo que podría constituir una subestimación de otros factores en el crecimiento económico (ver Arnold (2007), Cohen y Soto (2007), Aghion et al. (2010)).

El trabajo se divide en cinco secciones: la primera hace una breve revisión de la literatura reciente sobre capital humano y crecimiento; la segunda muestra los supuestos de un modelo de crecimiento básico del tipo de Solow (1957) pero donde el capital humano es parte de la función producción y se determina de la forma propuesta por Uzawa (1965) y Lucas (1988); la tercera determina, en ese mismo modelo, el porcentaje de tiempo dedicado al estudio a través de la maximización del valor presente del salario; la cuarta parte introduce la ecuación que hace endógeno el porcentaje del tiempo dedicado al estudio para obtener el crecimiento de largo plazo de la economía; finalmente, la última sección realiza el ejercicio primero de calibración y luego de simulación para México, en el cual se determina la influencia de diversos parámetros como la tasa de ahorro, la tasa de crecimiento de la población y variables relacionadas con la calidad de la educación en el crecimiento del capital humano y del producto per cápita de largo plazo de nuestro país.

\section{Breve revisión de la literatura reciente sobre capital humano y crecimiento económico}

Los efectos del capital humano sobre el crecimiento económico han sido estudiados desde hace muchos años. Desde el punto de vista teórico, los trabajos fundamentales en este tema son el de Uzawa (1965) y el de Lucas (1988). En términos empíricos algunos trabajos ya antiguos en el tema son los de Romer (1989), Barro (1991) y Mankiw, Romer y Weil (1992).

A partir del inicio del presente siglo, la discusión sobre el papel del capital humano en el crecimiento presenta dos posiciones: la primera es que si bien el capital humano incide sobre el crecimiento económico, no es el único factor y hay otros que pudieran explicar el crecimiento en igual o incluso en mayor medida.

La segunda posición defiende la tesis de que el efecto del capital humano se ha subestimado en las estimaciones de crecimiento económico y que, en el límite, dicho capital podría estar explicando una proporción mucho mayor del crecimiento observado de la que se creía antes.

\footnotetext{
8 Además de que si no se usan los instrumentos adecuados en las estimaciones éstas pueden ser sesgadas e inconsistentes.
} 
Describiremos los principales trabajos que pudieran catalogarse como de la primera posición:

Bils y Klenow (2000) en un análisis econométrico de corte transversal para 85 países analizan el efecto de la escolaridad sobre el crecimiento económico, encontrando que del total del crecimiento observado sólo alrededor de $30 \%$ se explica por la mayor escolaridad. La gran correlación que se encuentra entre capital humano y niveles de vida se debe, de acuerdo a estos autores, a una causalidad reversa, donde el crecimiento y el mayor ingreso per cápita son los que explican la creciente escolaridad.

Por su parte, Krueger y Lindahl (2004) en un estudio econométrico para cerca de 100 países encuentran que los mayores niveles de educación explican el crecimiento de países cuyo nivel de educación inicial es bajo, pero este efecto se llega a volver casi nulo en países con alta educación. ${ }^{9}$ En esta misma posición, Shapiro (2006) utiliza un ejercicio de calibración de una variante del modelo de Lucas (1988) para Estados Unidos, el cual investiga si los aumentos de productividad se deben a la mayor educación o mejoras en la calidad de vida de la población. Estos autores encuentran que aproximadamente el $60 \%$ del aumento de la productividad se debe a la mayor educación, pero el $40 \%$ se explica por mayores niveles de vida, los cuales están relacionados con mejores servicios de salud e infraestructura, entre otros factores.

Un artículo mucho más reciente sobre el tema es el de Aghion et al. (2010), que nuevamente muestra, en un ejercicio de corte transversal para muchos países, que el capital humano explica de manera considerable el crecimiento, pero que otros factores, como la salud, también tienen una influencia fundamental para explicar los mayores niveles de ingreso per cápita.

En otro artículo a nivel regional, Acemoglou y Dell (2010) analizan las diferencias entre los ingresos per cápita de distintos municipios de varios países de América Latina. Los autores encuentran que cerca de la mitad de dichas diferencias están explicadas por diferencias del capital humano. Sin embargo, la otra mitad de las diferencias se debe a cuestiones institucionales, geográficas e incluso culturales.

Entre los principales trabajos que defienden la segunda posición, la cual señala que es el capital humano el factor primordial que explica la mayor parte del crecimiento económico de largo plazo, los artículos seminales son el original de Uzawa (1965), el clásico de Lucas (1988) y el mucho más reciente del propio Lucas (2009). Este último encuentra apoyo en los trabajos antes publicados de Jones (2005) y Álvarez et al. (2008). ${ }^{10}$

La diferencia de la versión del artículo de Lucas de 2009 con el de 1988 radica en la forma en que se comporta el capital humano. En el artículo seminal

\footnotetext{
9 Estos resultados han sido criticados en el trabajo de Vandenbussche et al. (2004), quienes señalan que es necesario diferenciar el tipo de educación, por ejemplo primaria, secundaria y terciaria. Estimando una variante del modelo de Lucas (1988) estos autores encuentran que años adicionales de educación terciaria si tienen efecto en el crecimiento de países con niveles iniciales de educación altos pues amplían la frontera del conocimiento al incrementar la innovación.

10 Los autores son Álvarez, Buera y el propio Robert Lucas.
} 
de 1988 el crecimiento de la escolaridad es la que explica, en su totalidad, el crecimiento económico de largo plazo. En el artículo de 2009 la escolaridad es una condición necesaria pero no suficiente. Para que el motor de crecimiento sea permanente, es necesario que distintos tipos de capital humano interactúen en el trabajo (on the job). Sin esta interacción no hay crecimiento de largo plazo.

La tesis de que el capital humano explica una parte más grande del crecimiento económico de lo que se creía antes, ha ido ganando terreno también a nivel de estudios empíricos recientes:

Gennaioli et al. (2013) construyen una base de datos regional para más de 100 países. Al llevar a cabo estimaciones econométricas, estos autores encuentran que las diferencias de capital humano explican la mayor parte de las diferencias del producto per cápita de las regiones analizadas. No obstante, los autores insisten en la necesidad de separar el capital humano de los trabajadores y de los empresarios, los cuales, al menos a nivel teórico, tienen efectos diferentes sobre el crecimiento económico. Para mostrar lo anterior, los autores utilizan un ejercicio de calibración-simulación basado en los modelos de Lucas $(1988,2009)$. Su conclusión es que el nivel educativo de los empresarios es más importante que el de los trabajadores en la consecución de un mayor crecimiento económico. Por su parte, Manuelli y Seshadri (2014) plantean primero un modelo teórico que al calibrarlo les permite construir acervos de capital humano por país. El modelo también permite diferenciar la calidad del capital humano. Una vez que se lleva a cabo este análisis, el estudio econométrico de corte transversal muestra que el capital humano tiene una influencia muy elevada en la productividad factorial total y ésta, a su vez, en el crecimiento económico.

Además de la discusión relativamente reciente sobre el papel del capital humano en el crecimiento económico, hay otros sub temas en el tema más general de capital humano y crecimiento que están a discusión. Uno de ellos es el de la naturaleza del crecimiento económico, si se comporta más como lo explica Solow $(1956,1957)$ en un modelo de crecimiento exógeno, o está más relacionado con la explicación de Lucas $(1988,2009)$ de crecimiento endógeno. En estudios de panel para países de la OCDE, Arnold et al. (2007) y Cohen y Soto (2007) encuentran que la explicación de Lucas es más relevante que la de Solow para explicar el crecimiento económico.

Finalmente, para el caso de México hay poco estudios de la influencia del capital humano en el crecimiento económico. Mencionamos algunos de ellos:

Brock y Germán-Soto (2013) estiman una función producción para diferentes regiones de México. Esta función incluye al capital humano como uno de los factores relevantes. El resultado principal de este trabajo es que la apertura comercial que México ha experimentado desde los años ochenta modificó la estructura productiva. Antes de la apertura el impacto que tenían el capital físico y el capital humano en la producción era mayor que después de la apertura.

Un resultado tal vez congruente con el anterior es el de Atkin (2016). El artículo de este autor muestra que el crecimiento del sector manufacturero ha propiciado un cambio en la distribución de la educación, generando un aumento del abandono escolar, el cual surge del aumento en el costo de oportunidad de 
acceder a la escuela cuando se da la oportunidad de participar en el mercado laboral con una mayor demanda de empleos no calificados.

Por su parte, Ocegueda et al. (2013) utilizan un panel para estados de la república mexicana en el que analizan como incide la terminación de los grados escolares en el PIB estatal. El resultado principal es que los efectos de la educación primaria y secundaria son superiores a los de la educación terciaria y profesional, los cuales son incluso estadísticamente no significativos.

En otro artículo para México, Kido y Kido (2015) estiman una función de producción para México con datos anuales de 1980 a 2012. De acuerdo a estos autores, el capital humano tiene un efecto significativo sobre el producto pero sólo en largo plazo.

\section{El modelo de crecimiento básico}

El modelo parte de una función producción de bienes y servicios no educativos para una empresa típica. Esta función se basa en la propuesta por Lucas (1988):

$$
Y_{t}=K_{t}^{\alpha}\left(u h_{t} L_{b t}\right)^{1-\alpha} H_{t}^{\gamma}
$$

Donde

$Y:$ Producto total

$K$ : Capital físico

$L_{b}$ : Número de trabajadores en el sector productor de bienes y servicios no educativos.

$h$ : Capital humano dentro de la empresa

$H$ : Capital humano de la economía

$u$ : porcentaje del tiempo dedicado al trabajo en relación al tiempo total para estudiar y trabajar

$\alpha$ está entre 0 y 1

$\gamma \geq 0$

La función producción depende de los factores tradicionales: capital físico y trabajo. El factor que mide la productividad del trabajo es $u h$, donde $u$ es el tiempo que los trabajadores le dedican efectivamente al trabajo en la empresa y $h$ es el acervo de capital humano que poseen. La función producción considera la llamada externalidad de Lucas (1988) (el parámetro $\gamma$ de la función producción), por la cual el acervo de capital humano de todas las demás empresas de la economía tiene un efecto positivo sobre la producción de las empresas en particular.

En un mundo con empresas idénticas, o cuando menos muy similares, el capital humano de la economía es proporcional al capital humano de cada empresa. Así que es posible suponer $h=H$ sin pérdida alguna de generalidad.

En el sector educativo, la producción del flujo de capital humano que cada individuo obtiene toma la forma propuesta por Lucas (1988), la cual se basa en el trabajo original de Uzawa (1965):

$$
\frac{d h_{t}}{d t}=(1-u) \phi h_{t}
$$


El flujo de capital humano individual depende básicamente de tres argumentos: el porcentaje del tiempo que los trabajadores dedican al estudio $1-u$; la calidad de la educación, representada por el parámetro $\phi$, y el acervo de capital humano $h$. Uzawa (1965) considera que el parámetro $\phi$ está directamente relacionado con la razón de personas que trabajan en el sector educativo a las que trabajan en el sector de bienes $\left(L_{e} / L_{b}\right)$, donde $L_{e}$ es el número de personas que trabajan en el sector educativo. Lucas (1988) deja más libre el parámetro, de manera que además de incluir esa razón puede incluir otros factores, como los materiales de estudio y las tecnologías de aprendizaje, entre otros.

En términos per cápita, la función producción de bienes puede expresarse como $^{11}$

$$
y_{t}=k_{t}^{\alpha}\left(u h_{t}\right)^{(1-\alpha)} h_{t}^{\gamma}
$$

y: Producto per cápita $(Y / L)$

$k$ : Capital per cápita $(K / L)$

Asimismo, la acumulación de capital per cápita que surge del modelo original de Solow (Solow (1956),(1957)) se define como:

$$
\frac{\left(d k_{t}\right)}{d t}=s y_{t}-(\eta+\delta) k_{t}=s k_{t}^{\alpha}\left(u h_{t}\right)^{(1-\alpha)} h_{t}^{\gamma}-(\eta+\delta) k_{t}
$$

Donde $s$ es la tasa de ahorro de la economía, la cual se supone constante, $\eta$ es la tasa de crecimiento de la población y $\alpha$ es la tasa de depreciación del capital físico. Cuando el ahorro per cápita, representado por el término sy, es mayor que la depreciación del capital per cápita $(n+\delta) k$, el capital per cápita aumenta $(d k / d t>0)$.

Sustituyendo (3) en (4) y dividiendo toda la ecuación entre el capital per cápita, se obtiene.

$$
\begin{gathered}
\frac{d k_{t}}{d t} \frac{1}{k_{t}}=g_{k t}=\frac{s\left(u h_{t}\right)^{1-\alpha} h_{t}^{\gamma}}{k_{t}^{1-\alpha}}-(\eta+\delta) \\
\frac{\left(d g_{k t}\right)}{d t}<0
\end{gathered}
$$

Donde $g_{k}$ es la tasa de crecimiento del capital per cápita

Dado que la derivada del crecimiento del capital per cápita respecto al nivel de la misma variable es negativa, la ecuación diferencial (5) converge a un nivel de crecimiento estable del capital per cápita. Dicho crecimiento puede obtenerse igualando la tasa de crecimiento del numerador del cociente del primer término del lado derecho de la ecuación (5) con la tasa de crecimiento del denominador de dicho término, en cuyo caso y suponiendo que u es constante, se obtiene: ${ }^{12}$

\footnotetext{
11 En este caso se dividió la función producción por $L_{b}$, pero si $L_{e} / L_{b}$ es constante eso cualitativamente no afecta los resultados del modelo.

12 Para obtener la tasa de crecimiento del numerador y denominador del primer término del lado derecho de la ecuación (5), se toman los logaritmos de dichos términos y luego se derivan con respecto al tiempo. Como s y u se suponen constantes, los cambios de los logaritmos de esas variables en el tiempo son cero.
} 


$$
(1-\alpha+\gamma) g_{h}=(1-\alpha) g_{k}
$$

Siendo $g_{h}$ la tasa de crecimiento del capital humano.

La tasa de crecimiento del capital humano puede obtenerse dividiendo la ecuación (2) por el acervo de capital humano. El resultado de esta operación es

$$
g_{h}=\frac{d h_{t}}{d t} \frac{1}{h_{t}}=(1-u) \phi
$$

Si $u$ y $\phi$ son constantes, entonces la tasa de crecimiento del capital humano es también constante.

De aquí que por (6) y (7), se obtiene que

$$
g_{k}=\frac{(1-\alpha+\gamma)}{(1-\alpha)}(1-u) \phi
$$

La ecuación (8) muestra que el crecimiento del capital per cápita es proporcional al crecimiento del capital humano. El valor de la proporción sería exactamente igual a la unidad si la externalidad de Lucas fuera cero y mayor a uno si la externalidad realmente ocurre (es positiva).

Obteniendo logaritmos de la función producción (3) y derivando esos logaritmos

$$
g_{y}=\alpha g_{k}+(1-\alpha+\gamma) g_{h}
$$

Sustituyendo (7) y (8)

$$
g_{y}=\alpha \frac{(1-\alpha+\gamma)}{(1-\alpha)} g_{h}+\frac{(1-\alpha+\gamma)(1-\alpha)}{(1-\alpha)} g_{h}=\frac{(1-\alpha+\gamma)}{(1-\alpha)}(1-u) \phi=g_{k}
$$

En largo plazo, tanto el capital per cápita como el producto per cápita crecen a la tasa que muestra el lado derecho de la ecuación (8). Sin embargo, aun cuando se suponga que esa tasa es constante, no es posible saber cuál es su magnitud, pues tanto en el modelo de Uzawa (1965), como en el de Lucas (1988), el porcentaje de tiempo dedicado al estudio $(1-u)$, y por lo tanto también al trabajo, son variables endógenas. La tarea entonces es determinar una ecuación para el parámetro $u$ y de ahí definir cuál es la tasa de crecimiento del producto per cápita de equilibrio de largo plazo.

\section{La determinación del porcentaje de tiempo dedicado al trabajo y al estudio}

Los productores del sector de bienes maximizan beneficios. La función de beneficios es:

$$
K_{t}^{\alpha}\left(u h_{t} L_{t}\right)^{1-\alpha} h_{t}^{\gamma}-w_{t} L_{b t}-\left(r_{t}+\delta\right) K_{t}
$$

La maximización de esta función con respecto al capital físico y al trabajo $\left(K y L_{b}\right)$ implica que el producto marginal de los factores es igual al costo marginal de los factores: 


$$
\begin{gathered}
r_{t}+\delta=\alpha K_{t}^{\alpha-1}\left(u h_{t} L_{t}\right)^{1-\alpha} h_{t}^{\gamma}=\alpha k_{t}^{\alpha-1}\left(u h_{t}\right)^{1-\alpha} h_{t}^{\gamma} \\
w_{t}=(1-\alpha) K_{t}^{\alpha}\left(u h_{t} L_{t}\right)^{-\alpha}\left(u h_{t}\right) h_{t}^{\gamma}=(1-\alpha) k_{t}^{\alpha} u^{1-\alpha} h_{t}^{1-\alpha+\gamma}
\end{gathered}
$$

La ecuación (12) muestra que la productividad marginal del capital, que es la derivada de la función producción (1) con respecto al capital físico, es igual al costo marginal del capital, el cual se representa por la suma del rendimiento del capital $(r)$ y la tasa de depreciación del mismo. Por su parte, la ecuación (13) muestra que el salario real $(w)$ es igual al producto marginal del trabajo, el cual, a su vez, depende positivamente del capital per cápita, del porcentaje del tiempo dedicado al trabajo y del capital humano.

Para calcular $u$ suponiendo un estado estacionario permanente, o en vecindades muy cercanas a dicho estado, utilizamos los resultados de las ecuaciones (7) y (8), que implican que en el estado estacionario el capital físico per cápita y el capital humano crecen a las tasas señaladas por estas ecuaciones, de modo que las trayectorias del capital físico per cápita y el capital humano por individuo pueden definirse como: ${ }^{13}$

$$
\begin{gathered}
k_{t}=k(0) e^{\frac{(1-\alpha+\gamma)(1-u) \phi}{(1-\alpha)} t} \\
h_{t}=h(0) e^{(1-u) \phi t}
\end{gathered}
$$

Sustituyendo estas ecuaciones en la ecuación (13)

$$
w_{t}=(1-\alpha) u^{1-\alpha}\left(k(0) e^{\frac{(1-\alpha+\gamma)(1-u) \phi}{(1-\alpha)} t}\right)^{\alpha}\left(h(0) e^{(1-u) \phi t}\right)^{(1-\alpha+\gamma)}
$$

Esta expresión muestra que el porcentaje del tiempo dedicado al trabajo tiene dos efectos contrarios en el salario real a lo largo del tiempo: en el presente, un mayor porcentaje del tiempo dedicado al trabajo (mayor nivel de $u$ ) propicia un salario mayor. Sin embargo, conforme pasa el tiempo un mayor tiempo dedicado al estudio y un menor tiempo dedicado al trabajo aumenta el capital físico y el humano, y eso genera mayores pagos al trabajo. Lo anterior sugiere que hay un porcentaje óptimo de horas dedicado al trabajo, con el remanente dedicado al estudio.

La ecuación (14) se puede expresar como

$$
w_{t}=(1-\alpha) k_{0}^{\alpha} h_{0}^{1-\alpha+\gamma} u^{1-\alpha} e^{\frac{(1-\alpha+\gamma)}{(1-\alpha)}(1-u) \phi t}
$$

Los trabajadores desean maximizar el valor presente de sus pagos salariales. Supondremos para ello una tasa de interés constante de equilibrio en el estado

\footnotetext{
13 Hay que señalar que de acuerdo a los supuestos de este trabajo el crecimiento del capital humano individual toma el valor señalado en la ecuación (7) en todo momento del tiempo. Independientemente de si el capital físico per cápita está o no en su trayectoria de largo plazo.
} 
estacionario. También se hará el supuesto de un horizonte infinito de tiempo. De este modo, la función a maximizar es:

$$
\operatorname{Max} \int_{0}^{\infty} w_{t} e^{-r t} d t
$$

Utilizando las ecuaciones (15) y(17) esta integral se expresa como

$$
\int_{0}^{\infty} w_{t} e^{-r t} d t=(1-\alpha) k_{0}^{\alpha} h_{0}^{1-\alpha+\gamma} u^{1-\alpha} \int_{0}^{\infty} e^{-\left(r-\frac{(1-\alpha+\gamma)(1-u) \phi}{(1-\alpha)}\right) t}
$$

La misma integral puede expresarse como

$$
\begin{gathered}
\int_{0}^{\infty} w_{t} e^{-r t} d t=-\frac{(1-\alpha) k_{0}^{\alpha} h_{0}^{1-\alpha+\gamma} u^{1-\alpha}}{\left(r-\frac{(1-\alpha+\gamma)(1-u) \phi}{(1-\alpha)}\right)} \int_{0}^{\infty} e^{-\left(r-\frac{(1-\alpha+\gamma)(1-u) \phi}{(1-\alpha)}\right) t} \\
\left(-\left(r-\frac{(1-\alpha+\gamma)(1-u) \phi}{(1-\alpha)}\right)\right) d t
\end{gathered}
$$

La solución de la integral es

$$
\int_{0}^{\infty} w_{t} e^{-r t} d t=-\frac{(1-\alpha) k_{0}^{\alpha} h_{0}^{1-\alpha+\gamma} u^{1-\alpha}}{\left(r-\frac{(1-\alpha+\gamma)(1-u) \phi}{(1-\alpha)}\right)} e^{-\left(r-\frac{(1-\alpha+\gamma)(1-u) \phi}{(1-\alpha)}\right) t}
$$

La cual hay que evaluarla entre cero e infinito.

Para que la integral converja, es necesario que

$$
r-\frac{(1-\alpha+\gamma)(1-u) \phi}{(1-\alpha)}>0
$$

Esto implica claramente que la convergencia de la integral requiere que el rendimiento del capital sea mayor que la tasa de crecimiento del PIB per cápita, ya que el término que se está sustrayendo es efectivamente ese crecimiento (ver ecuación (8)). Recientemente, a raíz del trabajo de Piketty (2013), se ha generado una polémica sobre el tema del signo de la resta entre el rendimiento del capital y el crecimiento. En una gran cantidad de modelos económicos la desigualdad (22) es necesaria para la existencia de la solución del modelo. Sólo en casos como el modelo de Diamond (1965) (ver D. Romer (2006 capítulo 2 parte $\mathrm{B}$ )) puede ocurrir, aunque no necesariamente ocurre, lo contrario.

Si efectivamente la integral converge

$$
j=\int_{0}^{\infty} w_{t} e^{-r t} d t=\frac{(1-\alpha) k_{0}^{\alpha} h_{0}^{1-\alpha+\gamma} u^{1-\alpha}}{\left(r-\frac{(1-\alpha+\gamma)(1-u) \phi}{(1-\alpha)}\right)}
$$

Esta ecuación también se puede expresar como

$$
j=\frac{z u^{1-\alpha}}{\left(r-\frac{(1-\alpha+\gamma)(1-u) \phi}{(1-\alpha)}\right)} \quad z=(1-\alpha) k_{0}^{\alpha} h_{0}^{1-\alpha+\gamma}
$$


34 Nueva Época REMEF (The Mexican Journal of Economics and Finance)

Ahora de lo que se trata es de encontrar el valor de $u$ que maximice esta expresión

De aquí que

$$
\frac{d j}{d u}=\frac{z\left((1-\alpha) u^{-\alpha}\left(r-\frac{(1-\alpha+\gamma)(1-u) \phi}{(1-\alpha)}\right)-\frac{(1-\alpha+\gamma) \phi u^{1-\alpha}}{(1-\alpha)}\right)}{\left(r-\frac{(1-\alpha+\gamma)(1-u) \phi}{(1-\alpha)}\right)^{2}}=0
$$

Para que esta expresión sea igual a cero el numerador debe ser cero pues el denominador es distinto de cero. Por lo cual

$$
(1-\alpha) u^{-\alpha}\left(r-\frac{(1-\alpha+\gamma)(1-u) \phi}{(1-\alpha)}\right)-\frac{(1-\alpha+\gamma) \phi u^{1-\alpha}}{(1-\alpha)}=0
$$

$\mathrm{Al}$ dividir toda la ecuación por $u^{\alpha}$ y re arreglar términos se llega a

$$
(1-\alpha) r-(1-\alpha+\gamma)(1-u) \phi-\frac{(1-\alpha+\gamma) \phi u}{(1-\alpha)}=0
$$

Ésta es una ecuación lineal en $u$, cuya solución es

$$
u=\frac{(1-\alpha)}{\alpha(1-\alpha+\gamma)}\left((1-\alpha) \frac{r}{\phi}-(1-\alpha+\gamma)\right)
$$

En el caso particular del modelo de Solow, la externalidad de Lucas es igual a cero, así que $\gamma=0$. Entonces

$$
u=\frac{(1-\alpha)}{\alpha}\left(\frac{r}{\phi}-1\right)
$$

La proporción del tiempo dedicada al trabajo aumenta cuando la tasa de interés aumenta y disminuye cuando la calidad de la educación $\phi$ aumenta. La intuición es clara. Cuando la tasa de interés aumenta, dejar de trabajar de hoy tiene un costo de oportunidad más alto en términos del valor del ahorro perdido. Cuando la calidad de la educación aumenta, el salario esperado del futuro es más alto mientras más tiempo se le dedica a la educación, por lo mismo hay incentivos a dedicarle más tiempo a la educación y $u$ disminuye.

\section{El crecimiento endógeno de la economía}

Tomando la ecuación de la función de producción (3) y dividiéndola entre $u h_{t}$, da como resultado la función producción en términos de trabajo efectivo (ver por ejemplo Romer, (2006 p.11)).

$$
y_{a t}=k_{a t}^{\alpha} h_{t}^{\gamma}
$$

Donde

$$
y_{a t}=\frac{y}{u h_{t}}
$$




$$
k_{a t}=\frac{k}{u h_{t}}
$$

La ecuación diferencial del capital por unidad de trabajo efectivo es, utilizando la ecuación (7):

$$
\frac{d k_{a t}}{d t}=s k_{a t}^{\alpha} h_{t}^{\gamma}-\left(\eta+\delta+g_{h}\right) k_{a t}=s k_{a t}^{\alpha} h_{t}^{\gamma}-(\eta+\delta+(1-u) \phi) k_{a t}
$$

Donde $s k_{a} h^{\gamma}$ es el ahorro por unidad de trabajo efectivo y $\left(\eta+\delta+g_{h}\right) k_{a}$ es la depreciación del capital por unidad de trabajo efectivo. Siendo $\left(\eta+\delta+g_{h}\right)$ la tasa de depreciación del capital por unidad de trabajo efectivo. Cuando el ahorro por unidad de trabajo efectivo es mayor que la depreciación del capital en los mismos términos, el capital por unidad de trabajo efectivo aumenta $\left(d k_{a}>0\right)$.

Dividiendo la ecuación (31) por el capital por unidad de trabajo efectivo se obtiene

$$
\frac{d k_{a t}}{d t} \frac{1}{k_{a t}}=g_{k a t}=s \frac{h_{t}^{\gamma}}{k_{t}^{1-\alpha}}-(\eta+\delta+(1-u) \phi)
$$

Donde $g_{k a}$ es el crecimiento del capital por unidad de trabajo efectivo. Por las ecuaciones (7) y (8) es posible concluir que

$$
g_{k a t}=g_{k t}-g_{h t}=\frac{\gamma}{(1-\alpha)}(1-u) \phi
$$

De ahí que utilizando (32)

$$
\eta+\delta+(1-u) \phi\left(1+\frac{\gamma}{(1-\alpha)}\right)=s \frac{h_{t}^{\gamma}}{k_{t}^{1-\alpha}}
$$

Lo anterior implica entonces, despejando el capital por unidad de trabajo efectivo que

$$
k_{a t}=\left(\frac{s h_{t}^{\gamma}}{\eta+\delta+(1-u) \phi\left(1+\frac{\gamma}{(1-\alpha)}\right)}\right)^{\frac{1}{1-\alpha}}
$$

Por (30) y (35), la productividad marginal del capital puede expresarse como

$$
\begin{aligned}
\frac{d y_{a t}}{d k_{a t}} & =\alpha k_{a t}^{\alpha-1} h_{t}^{\gamma} \\
& =\alpha\left(\frac{s h_{t}^{\gamma}}{\eta+\delta+(1-u) \phi\left(1+\frac{\gamma}{(1-\alpha)}\right)}\right)^{-1} h_{t}^{\gamma} \\
& =\alpha\left(\frac{\eta+\delta+(1-u) \phi\left(1+\frac{\gamma}{(1-\alpha)}\right)}{s}\right)=r+\delta
\end{aligned}
$$


En equilibrio la productividad marginal del capital es igual al costo marginal del capital $r+\delta$

Utilizando la ecuación (28) también es cierto que

$$
r=\frac{\phi(1-\alpha+\gamma)}{(1-\alpha)}\left(\frac{\alpha u}{(1-\alpha)}+1\right)
$$

Las ecuaciones (36) y (37) resuelven simultáneamente para $r$ y $u$, las cuales quedan como formas reducidas en función de los demás parámetros. Una vez que se encuentran estas variables es posible conocer con precisión el crecimiento endógeno de la economía a través de las ecuaciones (8) y (10). Se repite la ecuación (10) por conveniencia:

$$
g_{y}=\alpha \frac{(1-\alpha+\gamma)}{(1-\alpha)} g_{h}+\frac{(1-\alpha+\gamma)(1-\alpha)}{(1-\alpha)} g_{h}=\frac{(1-\alpha+\gamma)}{(1-\alpha)}(1-u) \phi=g_{k}
$$

Un problema empírico de consideración es el de encontrar datos o alguna forma de resolver para el parámetro $\gamma$, la externalidad de Lucas. Por lo anterior, tomaremos en este artículo el caso donde $\gamma=0$, que está fuertemente relacionado con el modelo de Solow (1957) original.

Dado el supuesto anterior, la sustitución de (37) en (36) proporciona la siguiente forma reducida para $u$

$$
u=\frac{(1-\alpha)}{\alpha(s+(1-\alpha))}\left(\frac{1}{\phi}(\alpha(\eta+\delta)-s \delta)+(\alpha-s)\right)
$$

Por otra parte, y puesto que $\gamma=0$, en equilibrio de largo plazo

$$
g_{y}=g_{k}=g_{h}=(1-u) \phi
$$

Una vez que se endogeneiza el rendimiento del capital, la decisión de qué porcentaje del tiempo trabajar o estudiar depende de los parámetros del modelo: la tasa de ahorro $s$, la tasa de crecimiento de la población $\eta$, la tasa de depreciación del capital físico $\delta$, la calidad de la educación $\phi$ y la intensidad del capital como factor de producción $\alpha$. Es por esto que esta versión del modelo de Lucas (1988), como la original planteada por el mismo autor, constituyen modelos de crecimiento endógeno.

Dado que la variable $u$ es positiva, es claro por (39) que un incremento en la tasa de ahorro $s$ reduce el porcentaje de tiempo dedicado al trabajo $(u)$ y aumenta el crecimiento endógeno de la economía (ver ecuación (40)). También es claro que un aumento en la calidad de la educación $(\phi)$ tiene un efecto del mismo signo para $u$ y ampliado para el crecimiento de largo plazo (ver ecuaciones (39) y (40)). En cambio, un aumento de la tasa de crecimiento de la población $(\eta)$ aumenta el porcentaje de tiempo dedicado al trabajo y reduce el crecimiento económico de largo plazo.

\section{Calibración-simulación del modelo para el caso de México}

Una de las grandes ventajas de utilizar las ideas del modelo de Lucas en el contexto del modelo de Solow, y no en el contexto del modelo de optimización 
dinámica, es que es posible encontrar datos que directamente permiten calibrar el modelo para los países en particular. Muchos de estos datos son publicados por bases de datos como la que presentan Feenstra, Inklar y Timmer (2015) que es la nueva generación de la Penn World Table (PWT).

El objetivo principal de esta sección es analizar el efecto que tienen los distintos parámetros del modelo descrito en el crecimiento económico de largo plazo de una economía en particular, en este caso México. Para ello tomamos la ecuación de formación de capital humano, la ecuación de participación en el trabajo y la ecuación del rendimiento del capital. ${ }^{15}$

$$
\begin{gathered}
g_{h}=(1-u) \phi \\
u=\frac{(1-\alpha)}{\alpha(s+(1-\alpha))}\left(\frac{1}{\phi}(\alpha(\eta+\delta)-s \delta)+(\alpha-s)\right) \\
r=\phi\left(\frac{\alpha u}{(1-\alpha)}+1\right)
\end{gathered}
$$

También hacemos la consideración de largo plazo en la cual el crecimiento del producto per cápita y el capital físico per cápita es igual al crecimiento del capital humano.

$$
g_{y}=g_{k}=g_{h}
$$

En la PWT 8.1 es posible encontrar datos para la tasa de crecimiento de la población $\eta$. También contiene datos de la participación del trabajo en el producto (part), la cual en una función producción del tipo de Cobb-Douglas corresponde al parámetro $1-\alpha$. Por lo anterior, es posible considerar 1-part como proxy de la participación del capital en el producto $(\alpha)$. Asimismo, en el modelo de Solow $s$ es la tasa de ahorro, que por cuentas nacionales es igual a la inversión bruta entre el PIB. Por lo cual el dato histórico de razón de inversión a PIB también es un dato histórico de la tasa de ahorro total, doméstica y externa, observada. ${ }^{16}$

Por otra parte, la PWT 8.0 presenta datos para la tasa de depreciación del capital físico $\delta$. Faltan entonces datos para $u, g_{h} \mathrm{y} \phi$. Un indicador concreto de la calidad de la educación tal vez no exista. Tampoco existen datos de u como la proporción de horas dedicadas al trabajo, aunque en la PWT 8.1 hay datos del promedio de horas trabajadas anuales por empleado. Sin embargo, no hay datos directos de cuántas horas le dedica una persona promedio al estudio. ${ }^{17}$ Los datos que sí se encuentran son los del crecimiento del capital humano de una serie producida por Barro y Lee (2011), la cual también se publica en la PWT 8.1.

\footnotetext{
15 Todas bajo el supuesto de que $\gamma=0$.

16 Ex ante la tasa de ahorro y la razón de inversión a PIB planeadas pueden diferir si los parámetros que perciben ahorradores e inversionistas son diferentes. Sin embargo, ex post la tasa de ahorro total, doméstica y externa, es igual a la razón de inversión a PIB, por lo cual esta razón en el pasado constituye un dato histórico de la tasa de ahorro.

17 Esto quiere decir que de las horas que no se dedican al trabajo no es posible saber cuántas se dedican al estudio y cuántas al ocio y al descanso necesario.
} 


\subsection{Calibración del modelo}

En una primera instancia, el modelo se calibró utilizando los datos promedio de la PWT 8.1 entre 2000 y 2011. En este caso se tomaron los datos promedio de las variables $s, \alpha, \eta, \delta$ y $g_{h}$ y se encontraron como incógnitas $u, \phi$ y $r$ en las ecuaciones (41), (42) y (43). Cabe señalar que el crecimiento promedio observado del PIB per cápita de México entre 2000 y 2011 (0.7 geométrico y 1.1 aritmético) coincide en gran medida con el crecimiento observado promedio del índice de capital humano de la PWT 8.1 (1.0 tanto geométrico como aritmético). ${ }^{18}$ Lo que es muy congruente con los pronósticos del modelo de Lucas (1988) con externalidad igual a cero $(\gamma=0) .{ }^{19}$

Los datos que se obtuvieron de la PWT 8.0 y 8.1 y las incógnitas de calidad de la educación, $\phi$, rendimiento del capital $r$ y participación laboral $u$ en las ecuaciones (41) a (44) son los siguientes:

Cuadro 1. Calibración del Modelo Lucas-Solow para el caso de México

\begin{tabular}{|l|l|}
\hline $\begin{array}{l}\text { Variables obtenidas en la Penn World } \\
\text { Table }\end{array}$ & $\begin{array}{l}\text { Valores numéricos promedio entre 2000y } \\
2011\end{array}$ \\
\hline $\begin{array}{l}\text { Participación del capital en el producto } \\
\text { (proxy del parámetro } \alpha \text { ) }\end{array}$ & 0.614 \\
\hline $\begin{array}{l}\text { Relación de inversión a PIB (proxy de la tasa } \\
\text { de ahorro s) }\end{array}$ & 0.248 \\
\hline $\begin{array}{l}\text { Crecimiento de la serie de capital humano de } \\
\text { Barro y Lee (proxy de gh) }\end{array}$ & $0.01(1 \%)$ \\
\hline Tasa de depreciación del capital físico $\delta$ & $0.034(3.4 \%)$ \\
\hline Tasa de crecimiento de la población $\eta$ & $0.013(1.3 \%)$ \\
\hline Variables calibradas del modelo & \\
\hline Calidad de la educación ? & 0.048 \\
\hline Tasa de participación en el trabajo u & 0.789 \\
\hline Tasa de interés o de rendimiento del capital & $0.108(10.8 \%)$ \\
\hline
\end{tabular}

Fuente: PWT 8.0 y 8.1 y elaboración propia

\footnotetext{
18 Por promedio geométrico se entiende tomar la última cifra de la serie (en este caso en 2011), dividirla entre la primera cifra de la serie (en este caso en 2000), elevar ese factor a la razón de 1 entre el número de datos menos uno (en este caso 1/11), restarle 1 y multiplicar todo este factor por 100. En cambio el promedio aritmético consiste en obtener el promedio simple de los crecimientos anuales de la serie en cuestión.

19 La razón por la cual los promedios aritmético y geométrico de los crecimientos del PIB per cápita difieren mucho más que los mismos conceptos aplicados a la serie de capital humano es que la primera serie tiene mucha mayor volatilidad.
} 
Una vez que se obtuvieron estos datos, se tomó en cuenta el trabajo de Uzawa (1965) para modelar el parámetro $\phi$.

Uzawa (1965) supone un indicador de la calidad de la educación que está directamente relacionado con la razón de trabajadores de la educación a la fuerza laboral. Para México estos datos pueden encontrarse en dos bases estadísticas:

La base de datos de la Secretaría de Educación Pública $(\mathrm{SEP})^{20}$ provee la cantidad de maestros que hay a todos los niveles, desde educación primaria hasta posgrado. Estos datos son anuales entre 1990 y 2015.

Por otra parte, el INEGI, en su Banco de Información Económica (BIE), es la fuente oficial de datos de la población económicamente activa (PEA). Estos datos tienen periodicidad trimestral y se presentan desde 2005 hasta el 2015.

Una vez que se anualizaron estas últimas cifras, se tomó el promedio de la razón de maestros a población económicamente activa entre los años 2005 y 2011 para que fueran relativamente compatibles con los promedios de las otras variables de la PWT calculados entre 2000 y 2011.

De esta manera se hizo el supuesto de que el parámetro $\phi$ es proporcional a la razón de maestros a la PEA, de una manera muy similar a la que supone Uzawa (1965)

$$
\phi=\varphi_{1} R
$$

Donde $\psi_{1}$ es una constante y $R$ es la razón de maestros a la PEA. Como la $\mathrm{R}$ promedio entre 2005 y 2015 es 0.037 , el parámetro $\psi_{1}$ consistente con la calibración descrita es 1.29. Conviene señalar que el número total de maestros de todos los niveles educativos no constituye el número total de personas que ofrecen sus servicios en el sector educativo. Faltan funcionarios y otros empleados de la SEP que no son maestros, así como el personal administrativo y de servicio de todas las escuelas públicas y privadas. Ante la dificultad de obtener una cifra estadísticamente relevante para todos estas personas, se optó por tomar en cuenta sólo a los maestros.

\subsection{Simulación del modelo}

El ejercicio de simulación que se lleva a cabo pregunta lo siguiente:

Si el crecimiento del PIB per cápita, que entre 2000 y 2011 ha sido cercano a $1 \%$ promedio, quisiera elevarse al $2 \%$ en el largo plazo, ¿en cuánto tendrían que modificarse alguna, o algunas, de las siguientes variables: la tasa de ahorro $s$, o la tasa de crecimiento de la población $\eta$, o la razón de maestros a la PEAR?

Consideramos esas variables porque son las que en teoría serían sujetas de modificarse a través de políticas del gobierno. Difícilmente podrían modificarse la tasa de depreciación del capital $(\delta)$ o los coeficientes de la función producción $(\alpha) .^{21}$

20 Sistema Nacional de Información Estadística Educativa (SNIEE).

21 El gobierno puede modificar la tasa de depreciación contable, pero difícilmente puede modificar la tasa de depreciación física del capital. Algunas políticas en las cuales se regule el tiempo permitido para que funcione una máquina tal vez podrían afectar la tasa de depreciación del capital físico. 
En los años setenta el gobierno mexicano comenzó a hacer una gran campaña para reducir la tasa de crecimiento de la población. En esa época esa tasa era de $3.2 \%$, en la actualidad es cercana a $1.3 \%$. La reducción de la tasa pudo haber ocurrido tanto por políticas como por otras razones, por ejemplo la entrada al mercado de los anticonceptivos. Sin embargo, es muy probable que la actuación del gobierno sí haya tenido un impacto en el resultado final. En particular, en 1973 entró en vigor la Tercera Ley de General, la cual planteaba una estrategia global de reducción de la tasa de crecimiento de la población. A partir de entonces, el gobierno comenzó a proveer información sobre anticoncepción a nivel de los hospitales y clínicas públicas, así como a regalar o hacer que dichos anticonceptivos llegaran a la gente de forma gratuita, o cuando menos más barata. También hubo una política de llevar a cabo vasectomías gratuitas en los hospitales públicos, entre otras acciones (ver por ejemplo Córdoba (2011)).

Por otra parte, el gobierno podría modificar la tasa de ahorro básicamente a través de la política fiscal, la cual podría propiciar un aumento del ahorro público. Otras políticas, como la llamada bancarización de la economía, podrían incrementar la tasa de ahorro al expandir la oferta de servicios financieros en lugares del país donde éstos no existen, induciendo mayores niveles de ahorro e inversión. Sin embargo, la efectividad de dichas políticas se observaría muy probablemente en el largo plazo (ver por ejemplo Rozo y García (2011)).

En lo que respecta a la razón de maestros a PEA, una combinación de políticas que podría incrementar esta razón sería la de abrir más escuelas normales al tiempo que se mejoran los salarios relativos de los maestros. Con datos del BIE-INEGI es posible llevar a cabo un ejercicio para obtener el salario promedio personal del sector educativo en relación con el total de la economía. El resultado es que en 2005 dicha razón era de alrededor de 3.18, que implica que las personas que trabajan en el sector educativo en México están bastante mejor pagadas que las que laboran en actividades promedio de la economía. También es claro que dicha razón ha crecido, pues en 2011 era de 3.39 y llegó a subir hasta 3.55 en 2013. Dado que una proporción de cerca de $75 \%$ de los maestros de México trabajan en escuelas públicas, parece haber una preocupación del gobierno por mejorar sus condiciones laborales, lo que también explica porque la razón de maestros a PEA subió, entre 2005 y 2015, de $3.7 \%$ a 3.8\%. Incrementar todavía más el salario relativo de los maestros, junto con una mayor oferta de estudios para los normalistas, tendría tal vez un efecto positivo sobre la razón de maestros a PEA, aunque en este caso también eso llevaría tiempo (Uzawa, (1965).

El cuadro 2 muestra, en la columna (I), los promedios observados entre 2000 y $2011^{22}$ de la tasa de ahorro $s$, la tasa de crecimiento de la población $\eta$ y la razón de maestros a PEA. Esos promedios son consistentes con un crecimiento del capital humano y del PIB per cápita cercano a $1 \%$ anual, que es el promedio observado entre 2000 y 2011.

Las siguientes columnas (II), (III) y (IV) muestran resultados de diversos ejercicios de simulación:

222005 y 2011 para la razón de maestros a PEA 
La columna (II) muestra cuál debería ser la tasa de ahorro $(s)$, o la tasa de crecimiento de la población $(\eta)$, o la razón de maestros a PEA - tomando estos instrumentos por separado- para que el crecimiento promedio del PIB per cápita de la economía aumente del $1 \%$ observado entre 2000 y 2011 a $2 \%$ en el largo plazo.

La columna (III) muestra qué valor deberían tomar la tasa de ahorro $(s)$, la tasa de crecimiento de la población $(\eta)$ y la razón de maestros a PEA tomando una posible combinación de estos tres instrumentos- ${ }^{23}$ nuevamente para que el crecimiento promedio del PIB per cápita de la economía aumente del $1 \%$ observado entre 2000 y 2011 a $2 \%$ en el largo plazo.

La columna (IV) muestra qué valor deberían tomar la tasa de ahorro $(s)$, la tasa de crecimiento de la población $(\eta)$ y la razón de maestros a PEA-tomando una posible combinación de estos tres instrumentos ${ }^{24}$ para que el crecimiento del PIB per cápita de la economía aumente del 1\% observado entre 2000 y 2011 a $1.6 \%$ en el largo plazo.

Cuadro 2. Simulaciones del modelo: valor de los parámetros del modelo necesarios para alcanzar diversas tasas de crecimiento

\begin{tabular}{|c|c|c|c|c|}
\hline & (I) & (II) & (III) & (IV) \\
\hline & $\begin{array}{l}\text { Observados } \\
\text { (compatibles con 1\%) }\end{array}$ & $\begin{array}{l}\text { Utilizando un } \\
\text { solo parámetro } \\
\text { como } \\
\text { instrumento } \\
\text { (compatible con } \\
2 \%)\end{array}$ & $\begin{array}{l}\text { Utilizando una } \\
\text { combinación posible } \\
\text { de parámetros como } \\
\text { instrumentos } \\
\text { (compatible con } 2 \% \text { ) }\end{array}$ & $\begin{array}{l}\text { Utilizando una } \\
\text { combinación posible } \\
\text { de parámetros como } \\
\text { instrumentos } \\
\text { (compatible con } \\
1.6 \% \text { ) }\end{array}$ \\
\hline $\begin{array}{ll}\text { Tasa de } \\
\text { ahorro s }\end{array}$ & 0.248 & 0.33 & 0.29 & 0.26 \\
\hline $\begin{array}{l}\text { Tasa de } \\
\text { crecimiento } \\
\text { de la } \\
\text { población } \eta\end{array}$ & $0.013(1.3 \%)$ & 0.0 & $0.09 \quad(0.9 \%)$ & $0.09(0.9 \%)$ \\
\hline $\begin{array}{lr}\text { Razón } & \text { de } \\
\text { maestros } & \text { a } \\
\text { PEA R } & \end{array}$ & $\begin{array}{ll}0.037 & (3.7 \%)\end{array}$ & $0.049 \quad(4.9 \%)$ & $0.039 \quad(3.9 \%)$ & $0.039(3.9 \%)$ \\
\hline
\end{tabular}

Fuente: Primera columna (I) datos de la PWT 8.1, BIE del INEGI y Estadísticas de la SEP. Siguientes columnas (II), (III) y (IV) cálculos del autor utilizando el modelo descrito con datos del crecimiento de la población obtenidos de la CONAPO.

Los valores de la columna (II) del cuadro 2 sugieren que sería casi imposible utilizar un solo parámetro para aumentar el crecimiento del PIB per cápita y

23 Claramente podría haber una infinita combinación de valores de los instrumentos para lograr que el crecimiento promedio del PIB per cápita aumente de $1 \%$ a $2 \%$, lo que se está considerando es una combinación razonable de valores para esos instrumentos. La racionalidad de esa combinación viene explicada más adelante en el texto principal.

24 Mismo comentario que la nota de pie de página anterior. 
del capital humano de $1 \%$ a $2 \%$ anual. Así por ejemplo, el ahorro tendría que aumentar 8 puntos del PIB, lo que a precios actuales implica un incremento de alrededor de 1.4 billones de pesos o de 80 mil millones de dólares de ahorro adicional por año. Si se utilizara sólo la política de población, habría que reducir la tasa actual de crecimiento de la población a cero. Sin embargo, el Consejo Nacional de Población (CONAPO) prevé que en los próximos diez años la tasa promedio se reduzca sólo a $0.9 \%$. Utilizar la razón de maestros a PEA requeriría aumentar más de medio millón de maestros a la educación escolarizada, algo muy poco factible de lograr en un mediano plazo.

Por otra parte, la columna (III) muestra que considerando los pronósticos de CONAPO sobre población de crecimiento de $0.9 \%$ promedio en los próximos años, y el supuesto relativamente factible de aumentar la razón de maestros a PEA en 0.1 puntos porcentuales ${ }^{25}$ que dado el valor de la PEA actual significan alrededor de 50000 personas, la tasa de ahorro tendría que aumentar de $24.8 \%$ a $29 \%$ del PIB. Este valor es de alrededor de 0.7 billones de pesos o de cuarenta mil millones de dólares anuales. Parece difícil lograrlo más aún cuando el promedio de $24.8 \%$ del PIB entre 2000 y 2011 está compuesto por datos que muestran una tendencia a la baja de la tasa de ahorro.

Un escenario aparentemente más factible se presenta en la columna (IV). Aquí se supone que la tasa de ahorro sube a $26 \%$, mientras que el crecimiento de la población y la razón de maestros a PEA permanecen como en la columna (III). En este caso el ahorro tendría que aumentar alrededor de 0.2 billones de pesos actuales, los cuales son aproximadamente 12 mil millones de dólares. A su vez, habría que introducir alrededor de 50000 maestros más al sector educativo. La tendencia que lleva este rubro permite prever que esto sea posible.

El efecto de un incremento de la tasa de ahorro de $24.8 \%$ promedio a $26 \%$ promedio, una caída de la tasa de crecimiento de la población de 1.3\% a $0.9 \%$ y un aumento de la razón de maestros a la PEA de $3.8 \%$ a $3.9 \%$ propicia un incremento proyectado del crecimiento de largo plazo del PIB per cápita de $1 \%$ a 1.6\%. ¿Cuál sería el principal problema para lograr este resultado? Muy probablemente el aumentar la tasa de ahorro.

El cuadro 3 muestra el resultado para la tasa de crecimiento del PIB per cápita, el porcentaje del tiempo dedicado al trabajo u y el rendimiento del capital r compatibles con las columnas (I), (III) y (IV) del cuadro 2. La columna (I) del cuadro 3 muestra los valores observados o calibrados para las variables ya descritas en este párrafo. La columna (II) muestra los resultados de estos mismos valores con los supuestos de la columna (III) del cuadro 2. A su vez, la columna (III) del cuadro 3 muestra esos mismos valores con los supuestos de la columna (IV) del cuadro 2.

25 El promedio de la razón de maestros a PEA entre 2005 y 2011 es de 3.7\%. No obstante, para 2014 el valor observado de la variables subió a $3.8 \%$. 
Cuadro 3. Valor de las variables endógenas del modelo en dos escenarios simulados

\begin{tabular}{|l|l|l|l|}
\hline & \multicolumn{1}{|c|}{ (I) } & \multicolumn{1}{|c|}{ (II) } \\
\hline & $\begin{array}{l}\text { Resultados compatibles } \\
\text { con los supuestos de la } \\
\text { columna (I) del cuadro 2 }\end{array}$ & $\begin{array}{l}\text { Resultados compatibles } \\
\text { con los supuestos de la } \\
\text { columna (III) del cuadro } \\
2\end{array}$ & $\begin{array}{l}\text { Resultados compatibles } \\
\text { con los supuestos de la } \\
\text { columna (V) del cuadro 2 }\end{array}$ \\
\hline $\begin{array}{l}\text { Crecimiento del PIB per } \\
\text { cápita }\end{array}$ & $0.01(1 \%)$ & $0.02(2 \%)$ & $0.016(1.6 \%)$ \\
\hline $\begin{array}{l}\text { Porcentaje del tiempo } \\
\text { dedicado al trabajo }\end{array}$ & 0.789 & 0.608 & 0.685 \\
\hline Rendimiento del capital & 10.7 & 9.9 & 10.5 \\
\hline
\end{tabular}

Fuente: Elaborado por el autor con el modelo descrito.

Un resultado que salta a la vista es el del porcentaje del tiempo dedicado al trabajo. Para aumentar el crecimiento de largo plazo ese porcentaje debe caer sustancialmente. Si se dedicaran 10 horas de lunes a viernes al trabajo y al estudio, el valor de u calibrado en el modelo señalaría que se utilizan casi 8 horas para trabajar y 2 para estudiar (ver columna (I) cuadro 3 la cifra 0.789). Para lograr un crecimiento del $2 \%$ anual en el largo plazo, habría que dedicar casi 6 horas a trabajar y 4 a estudiar (ver columna (II) del cuadro 3 la cifra 0.608). Para lograr $1.6 \%$ de crecimiento habría que dedicar casi 7 horas a trabajar y 3 a estudiar (ver columna (III) del cuadro 3 la cifra de 0.685). El modelo de Lucas (1988) supone total flexibilidad de los trabajadores para escoger su tiempo de trabajo y estudio, algo que no sucede siempre en el mundo real. Sin embargo, en la vida ordinaria no necesariamente hablamos de una sola persona tomando decisiones, sino de que más personas decidan estudiar en vez de trabajar, con lo cual reducir una hora promedio de trabajo al día podría tal vez ser factible.

Otro punto importante a considerar es que en el modelo de Lucas el aumento del crecimiento a través de personas que estudian más y, por ende, trabajan menos puede tener un costo elevado en el corto plazo. Si bien es cierto que el crecimiento de largo plazo aumenta, en el corto plazo la reducción de horas de trabajo reduce, ceteris paribus, el PIB total y el PIB per cápita (ver ecuaciones 1 y 3 , si el parámetro u cae esas variables también caen). Eso hace difícil la decisión de política económica de lograr un mayor crecimiento en el futuro. 
44 Nueva Época REMEF (The Mexican Journal of Economics and Finance)

\section{Conclusiones}

Este trabajo presenta una metodología para llevar a cabo una calibración del modelo de crecimiento de Lucas (1988) utilizando como marco de referencia una versión del modelo de Solow (1957).

En términos del método, el trabajo plantea la maximización del valor presente de los salarios reales para encontrar la relación de tiempo óptima entre trabajo y estudio $(u)$. La ventaja de este método es que cuando se lleva a cabo en el estado estacionario permite una solución lineal del problema. Una vez que se obtiene la ecuación óptima del parámetro $u$, es posible encontrar el crecimiento del PIB per cápita de largo plazo, la relación mencionada $(u)$ y el rendimiento del capital como incógnitas.

La utilidad de la metodología descrita es que aplicándola en un modelo del tipo de Solow permite utilizar muchos datos observados para hacer simulaciones. La misma metodología se podría aplicar en un modelo del tipo de Ramsey, pero en este caso habría que estimar econométricamente parámetros como la tasa de descuento intertemporal y la elasticidad de sustitución en el consumo. Esto, como se discutió en el texto, no siempre es fácil.

Una limitación de la metodología es que en estricto sentido sólo es útil para evaluar estados estacionarios. Generalizarla para cualquier circunstancia requeriría de un manejo matemático muy complejo. Sin embargo, con cambios muy pequeños alrededor del estado estacionario sería posible utilizar la misma ecuación del parámetro $u$ y se podría simular una transición al siguiente estado estacionario como una aproximación lineal. Esto sería recomendable hacerlo en un trabajo futuro.

Aplicando el método descrito al caso de México, los resultados muestran que la tarea de elevar el crecimiento de largo plazo en sólo 0.6 décimas de punto porcentual por año requiere de alrededor de 12 mil millones de dólares actuales anuales de incremento en el ahorro, o 1.2 puntos del PIB. Este efecto se podría lograr en un tiempo relativamente corto, sin sacrificar fuertemente el consumo doméstico, porque el déficit en cuenta corriente como porcentaje del PIB en México está aumentando, lo que implica un incremento del ahorro externo. Sin embargo, la historia señala un fuerte riesgo de aumentar el crecimiento de esa forma pues ello va aparejado a un aumento considerable de la deuda externa. Las crisis macroeconómicas de 1982-1983 y 1994-1995 fueron antecedidas por niveles crecientes de déficit en cuenta corriente que se volvieron insostenibles. Todo esto indica que probablemente en el futuro cercano el crecimiento económico de México seguirá siendo bajo.

Posiblemente las contribuciones más importantes de este trabajo sean las siguientes:

Primero, la de mostrar que las ideas centrales de Lucas (1988) pueden aplicarse en contextos distintos a los planteados por el propio Lucas $(1988,2009)$ y seguidores. ${ }^{26}$ La ventaja de esto es que los datos para calibrar y simular esta variante del modelo de Lucas son mucho más accesibles que los que se necesitan para hacer un ejercicio similar utilizando el modelo original. Por ejemplo, con la metodología propuesta se podrían hacer, con relativa facilidad, ejercicios como

26 Por ejemplo, Gennaioli et al. (2013) y Manuelli y Seshadri (2014). 
el del presente trabajo para decenas de países sólo con los datos publicados por la PWT. ${ }^{27}$ En cambio, si se utilizara la metodología original propuesta por Lucas (1988), o alguna variante (Lucas (2009)), el trabajo econométrico anterior sería enorme y la tarea de calibrar, simular y hacer comparaciones entre países se volvería sumamente difícil.

Segundo, la de calibrar y simular la variante del modelo de Lucas (1988) para México, algo que hasta donde tenemos noticia nadie ha hecho y que, además, permite relacionar el capital humano y su crecimiento con la tasa de ahorro, la tasa de crecimiento de la población y la razón de maestros a PEA. Eso es una ventaja en relación con muchos modelos econométricos que a partir de modelos de crecimiento exógeno generan un divorcio ex ante entre el capital humano y las otras variables que determinan el crecimiento.

Tercero, que las simulaciones del modelo de Lucas para México permiten determinar, con cierto grado de confianza, que desafortunadamente el crecimiento económico de México seguirá siendo bajo en los próximos años.

\section{Bibliografía}

Acemoglou, D. (2010). Productivity Differences Between and Within Countries. American Economic Journal, Macroeconomics, 2, 169-188.

Aghion, P, P. Howitt \& F. Murtin. (2010). The Relationship Between Health and Growth: When Lucas Meets Nelson-Phelps. NBER Working Paper 15813. National Bureau of Economic Research. Cambridge, Massachusetts.

Alvarez, F, Buera, F \& R. Lucas (2008). Models of Idea Flows. NBER Working Paper 14135. National Bureau of Economic Research. Cambridge, Massachusetts.

Arnold, J, A. Bassanini \& S. Scarpetta. (2007). Solow or Lucas? Testing Growth Models Using Panel Data From OECD Countries. OECD Economics Department Working Papers No 592.

Arrow, K (1962). The Economic Implications of Learning by Doing. Review of Economic Studies, 29, 155-173.

Atkin, D. (2016). Endogenous Skill Acquisition and Export Manufacturing in Mexico. American Economic Review, 106, 2046-2085.

Attanasio, O \& H. Low (2002). Estimating Euler Equations. The Institute for Fiscal Studies. Working Paper 02/06. London, England

Barro, R (1991). Economic Growth in a Cross Section of Countries. Quarterly Journal of Economics, 106, 407-444.

Barro, R (2001). Human Capital and Growth. American Economic Review, 91, 12-17.

Barro, R \& Lee J. (2011). A New Data Set of Educational Attainment in the World 19502010. http://www.barrolee.com/papers/Barro_Lee_Human_Capital_Update_2011Nov. p df. Consultado en diciembre de 2015.

Bils, M \& Klenow P. (2000). Does Schooling Cause Growth? American Economic Review, 90, 1160-1183.

Brock, G \& German-Soto V. (2013). Regional Industrial Growth in Mexico: Do Human Capital and Infrastructure Matter? Journal of Policy Modeling, 35, 228-242.

Cass, D (1965). Optimal Growth in an Aggregative Model of Capital Accumulation. Review of Economic Studies, 37, 233-240.

27 Una parte que sería muy difícil de llevar a cabo para muchos países sería la de modelar la calidad de la educación como función de la razón de maestros a PEA, como si se hizo en este trabajo para el caso de México. Sin embargo, aun así, el parámetro de calidad de educación puede obtenerse con la calibración del modelo y lo que sí se podría hacer para muchos países sería analizar los efectos en el crecimiento de largo plazo y el crecimiento del capital humano de cambios en la tasa de ahorro y cambios en la tasa de crecimiento de la población. 
Cohen, D y Soto M. (2007). Growth and Human Capital: Good Data Good Results. Journal of Economic Growth, 12, 51-76.

Consejo Nacional de Población (CONAPO) (2015). Proyecciones de Población 2010-2050 en México. http://www.conapo.gob.mx/es/CONAPO/Proyecciones Consultado en diciembre de 2015 .

Cooper, R, Haltinwanger J, \& Willis M. (2010). Euler-Equation Estimation for Discrete Choice Models: A capital Accumulation Application. The Federal Reserve Bank of Kansas City. Research Working Paper 10-04. Kansas City.

Córdoba, D (2011). El control demográfico en México. Revista de Especialidades Médico Quirúrgicas, 16, 1-2. Instituto Nacional de Seguridad y Servicios Sociales de los Trabajadores del Estado de México.

Dao, M.Q (2012). Population and Economic Growth in Developing Countries. International Journal of Academic Research in Business and Social Sciences, 2, 6-17.

Diamond, P (1965). National Debt in a Neoclassical Growth Model. American Economic Review, 55, 1126-1150.

Feenstra, R., Inklaar R \& Timmer M. (2015). The Next Generation of the Penn World Table. American Economic Review. Puede obtenerse a través de www.qqdc.net/pwt

Fuhrer, J. \& Rudebusch G. (2004). Estimating the Euler Equation for Output. Journal of Monetary Economics, 51, 1133-1153.

Fuhrer, J. C \& Olivei P. (2004). Estimating Forward Looking Euler Equations with GMM Estimators. Federal Reserve Bank of Boston. Working Paper 04-2. Boston, Massachusetts.

Gennaioli, N, La Porta, R. López de Silanes F, \& Shleifer A. (2013). Human capital and regional development. Quarterly Journal of Economics, 10, 105-164.

Instituto Nacional de Estadística, Geografía e Informática (INEGI) (2015). Banco de Información Económica (BIE). http://www.inegi.org.mx/sistemas/bie/ Consultado en diciembre de 2015 .

Kido A, \& Kido M. (2015). Modelos teóricos del capital humano y señalización: un estudio para México. Contaduría y Administración, 60, 723-734

Koopmans, T (1965). On the Concept of Optimal Economic Growth. The Econometric approach to development planning. Amsterdam, North Holland.

Kruger A. \& Lindahl M. (2001) Education for growth: Why and for whom? Journal of Economic Literature, 39, 1101-1136.

Lewbel A, Linton O \& Srisuma S. (2010). Non Parametric Euler Equation Identification and Estimation.. Mimeo. Puede encontrarse en https://www2.bc.edu/ lewbel/nonparameu lerest.pdf. Consultado en diciembre de 2015.

Lucas, R (1988). On the Mechanism of Economic Development. Journal of Monetary Economics, 22, 3-42.

Lucas, R (2009). Ideas and growth. Economica, 76, 1-19.

Mankiw G, Romer D. \& Weil D. (1992). A Contribution to the Empirics of Economic Growth. Quarterly Journal of Economics, 107, 407-437.

Manuelli R. \& Seshadri A. (2014). Human Capital and the Wealth of Nations. American Economic Review, 104, 726-762

Ocegueda J, Meza F. \& Coronado M. (2013). Impacto de la educación en el crecimiento económico de México, 1990-2008. Revista Internacional Administración en Finanzas, $6,75-88$

Penn World Table 8.1. Robert Feenstra, Robert Inklaar y M. Timmer. http://www.rug.nl/ research/ggdc/data/pwt/pwt-8.1 Consultada en diciembre de 2015.

Piketty, T (2014). El Capital en el Siglo XXI. 1 ed. Fondo de Cultura Económica, Ciudad de México.

Rebelo, S (1992). Long-Run Policy Analysis and Long-Run Growth. Journal of Political Economy, 99, 500-521.

Rodríguez-Arana, A (2012). Estimación de una Ecuación de Euler del Consumo per Cápita para México: 1980-2010. Revista Mexicana de Economía y Finanzas. Nueva Época (REMEF), 7, 27-48. 
Romer, D (2006). Macroeconomía Avanzada. 3 ed. McGraw Hill. Madrid.

Romer, P. (1986). Increasing Returns and Long Run Growth. Journal of Political Economy, 94, 1002-1037.

Romer, P. (1989). Human capital and growth: Theory and evidence. National Bureau of Economic Research Working Paper No 3173. Cambridge, Massachusetts.

Rozo, C. \& García, J. (2011). Bancarización en México. En La UAM ante la sucesión presidencial. Propuestas de política económica y social para el nuevo gobierno. Federico Novelo (editor). Universidad Autónoma Metropolitana, Plantel Xochimilco.

Secretaría de Educación Pública. (2015) Sistema Nacional de Educación Estadística Educativa (SNIE). http://www.snie.sep.gob.mx/estadisticas_educativas.html Consultado en diciembre de 2015.

Solow, R (1956). A Contribution to the Theory of Economic Growth. Quarterly Journal of Economics, 70. 65-94.

Solow, R (1957). Technical Change and the Aggregate Production Function. Review of Economic and Statistics, 39, 312-320.

Shapiro, J. (2006). Quality of Life, Productivity and the Growth Effects of Human Capital. Review of Economics and Statistics, 88, 324-335.

Uzawa, H (1965). Optimal Technical Change in an Aggregative Model of Economic Growth. International Economic Review, 6-1, 18-31.

Vandenbussche P., Aghion A., \& Meghir, C. (2004). Growth, Distance to Frontier and Composition of Human Capital. Journal of Economic Growth 